\title{
Molecular Gas and Star Formation in BIMA SONG Bars
}

\author{
Kartik Sheth \\ Department of Astronomy, U. Maryland, College Park, MD 20742-2421 \\ S.N. Vogel, A.I. Harris (U. Maryland), M.W. Regan (STScI), M.D. \\ Thornley (NRAO), T.T. Helfer, T. Wong, L. Blitz, D.C-J. Bock \\ (U.C.-Berkeley)
}

\begin{abstract}
Using a sample of 7 barred spirals from the BIMA Survey of Nearby Galaxies (SONG), we compare the molecular gas distribution in the bar, to recent massive star formation activity. In all 7 galaxies, $\mathrm{H} \alpha$ is offset azimuthally from the $\mathrm{CO}$ on the downstream side. The maximum offset, at the bar ends, ranges from $170-570 \mathrm{pc}$, with an average of $320 \pm 120$ pc. We discuss whether the observed offsets are consistent with the description of gas flows in bars provided by the two main classes of models: n-body models and hydrodynamic models.
\end{abstract}

1. Star Formation in Bars: How and why do stars form where they do ? To answer this question one may begin by studying star formation activity and its molecular gas environment in a variety of environments, such as bars, spiral arms, and rings. Then by drawing together physical processes and triggers that are common in these different regions, we may shed light on how star formation might occur. Here we concentrate on bars in $7 \mathrm{SAB}$ galaxies from the BIMA Survey of Nearby Galaxies (SONG). Though classified as weak bars, some may be strongly barred, as shown by recent near-infrared surveys [1]. We compare the molecular gas distribution, traced by the CO $(\mathrm{J}=1-0)$ emission line, to the star formation activity traced by the $\mathrm{H} \alpha$ emission line. While overlays of $\mathrm{CO}$ and $\mathrm{H} \alpha$ maps show qualitative agreement, we use a cross-correlation analysis to quantify the relationship between the two.

2. The Cross-Correlation Process: After matching the images in resolution, we deproject them and convert them to polar coordinates. To cross-correlate the images, we overlay and shift one image against the other, at each step multiplying the images pixel by pixel and recording the sum. The offset lag at which the two images match best has the largest correlation value. The results of our analysis are shown in Figure 1.

\section{Results: Interpreting in Context of Gas Flow Models:}

- In all 7 galaxies, $\mathrm{H} \alpha$ is predominantly offset azimuthally from the CO. Moreover, the offset is always on the downstream side.

- Maximum offset (measured at bar ends): 170-570 pc, Mean: 320 \pm 120 pc.

3a. N-body simulations: Gas clouds behave as in spiral arms, crowding in the dust lane, but eventually crossing it on the leading side [2]. These models 

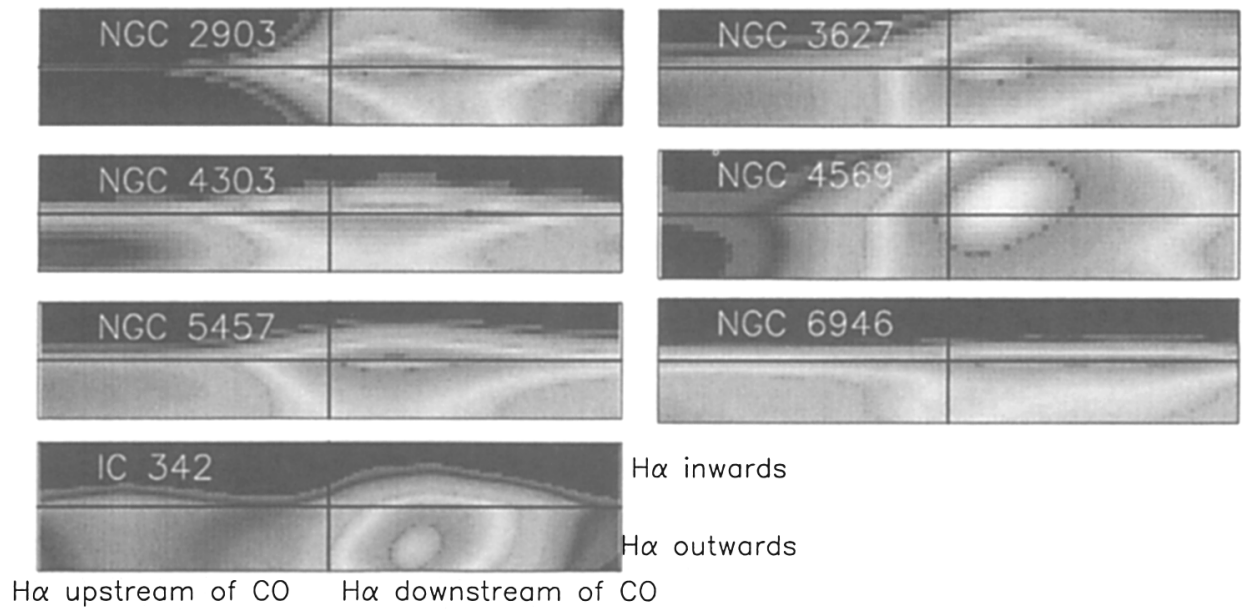

Figure 1. $\mathrm{H} \alpha-\mathrm{CO}$ cross-correlation for all 7 bars. Above (Below) the cross-hair indicates a radial offset of $\mathrm{H} \alpha$ inwards (outwards) from the CO. Left (Right) of the cross-hair indicates an azimuthal offset with $\mathrm{H} \alpha$ upstream (or downstream) of CO.

predict the observed offset of $\mathrm{CO}$ and $\mathrm{H} \alpha$ but fail to reproduce the straight dust lanes and high shear across the dust lanes. So these models are not applicable in all cases.

3b. Hydrodynamic simulations: Gas undergoes a hydrodynamic shock at the dust lane, and flows down the dust lane, never crossing the dust lane. Though the dust lane is inhospitable for star formation [3], stars may form in dust spurs upstream of dust lane [4]. Since none of the 7 galaxies show offsets $>500 p c$, star formation may occur in dust spurs; the distance between the spurs and HII regions is consistent with typical gas speeds $(50 \mathrm{~km} / \mathrm{s})$ and HII region lifetimes. The offset also indicates that HII regions spend most of their time on the leading side of the dust lane, thus constraining star formation to occur very close $(100-200 \mathrm{pc})$ to the dust lane. The dust lane shock may be involved, if not in triggering the star formation, in formation of dust spurs.

4. Conclusions: $\mathrm{H} \alpha$ emission is predominantly offset downstream from bar dust lanes; the offset is consistent with both sets of gas flow models. Larger sample size with stellar kinematics information is necessary for distinguishing between the models. This work supported by NSF AST-9981289, AST-9981308. Poster URL: http://bima.astro.umd.edu/projects/bimasong/pubs/iau205poster.ps References

[1] Mulchaey, J.S., Regan, M.W., \& Kundu, A. 1997, ApJS, 110, 299

[2] Combes, F., \& Gerin, M. 1985, A\&A, 150, 327

[3] Reynaud, D., \& Downes, D. 1998, A\&A, 337, 671

[4] Sheth, K., Regan, M.W., Vogel, S.N. \& Teuben, P.J. 2000, ApJ, 533, 221 